\title{
Affective infrastructures: toward a cultural neuropsychology of sport
}

\author{
Leslie L. Heywood ${ }^{1,2 *}$ \\ 1 Institute for Evolutionary Studies, Binghamton University, Binghamton, NY, USA \\ ${ }^{2}$ Department of English, Binghamton University, Binghamton, NY, USA
}

\author{
Edited by: \\ Melanie L. Shoup-Knox, University of \\ Pittsburgh, USA \\ Reviewed by: \\ Paul M. Nealen, Indiana University of \\ Pennsylvania, USA \\ Justin R. Garcia, Binghamton \\ University, USA \\ *Correspondence: \\ Leslie L. Heywood, Department of \\ English, Binghamton University, P.O. \\ Box 6000, Binghamton, NY \\ 13902-6000, USA. \\ e-mail: heywood@binghamton.edu
}

Recently there has been a turn toward considerations of embodiment, cognition, and context in sport studies. Many researchers have argued that the traditional focus on clinical psychology and performance enhancement within the discipline is incomplete, and now emphasize the importance of athletes' social and familial contexts in a research paradigm that examines interconnections between movement, cognition, emotion, and the social and cultural context in which movement takes place. While it is important that the sport studies focus is being expanded to consider these interactions, I will argue that this model is still incomplete in that it is missing a fundamental variable - that of our evolutionary neurobiological roots. I will use the work of affective neuroscientists Jaak Panksepp and Stephen Porges to show that because sport so clearly activates neural systems that function at both proximate and ultimate levels of causation, it can be seen to serve fundamental needs for affective balance. A neurobiology of affect shows how the evolution of the mammalian autonomic nervous system has resulted in neurophysiological substrates for affective processes and stress responses, and has wide-ranging implications for sport studies in terms of suggesting what forms of coaching might be the most effective in what context. I propose the term cultural neuropsychology of sport as a descriptor for a model that examines the relationships between neurophysiological substrates and athletes' social and familial contexts in terms of how these variables facilitate or fail to facilitate athletes' neuroceptions of safety, which in turn have a direct impact on their performance. A cultural neuropsychological model of sport might thereby be seen to elaborate a relationship between proximate and ultimate mechanisms in concretely applied ways.

Keywords: affective neuroscience, SEEKING/PLAY systems, neuroception, sport studies, evolutionary neurobiology

\section{INTRODUCTION: WHAT AN EVOLUTIONARY PERSPECTIVE ON AFFECT ADDS TO SPORT STUDIES}

While sport sociology has tended to focus on the large, macro-level, culturally derived aspects of sport and physical activity such as the economics of sport, the ways sport contributes to nationalistic ideologies, and the politics of gender, sport psychology and kinesiology have tended to focus on the micro-level aspects of clinical psychology and performance enhancement for the individual athlete (Hanrahan and Anderson, 2010). Both of these models exclude an embodied theory of the emotions. While sport sociology, psychology, and kinesiology have contributed to a complicated, variable, and deeply informative body of work on sport, an evolutionary perspective on the emotions can provide us with the most comprehensive model to date, a model that would truly integrate ultimate and proximate-level analysis. This perspective provides an analysis of the neurological mechanisms that determine how our phylogenetically modified autonomic nervous systems (ANS) respond to environmental challenges, thus giving us a model of the interaction between our psychological experiences and our physiological regulation. A consideration of this interaction unifies sociological, psychological, and neuroscientific analysis in an evolutionary framework that leads to unique conclusions about the ways sport and physical activity function culturally, socially, and individually. Conversely, sport and physical activity can serve as model systems for the demonstration of evolutionary principles, "walking the walk to teach the talk" (Platek et al., 2011).

Previously, when emotion has been studied in the context of sport, it has been largely relegated to the realm of performance enhancement. Yuri Hanin's Emotions in Sport, for instance, employs what he calls an "Individual Zones of Optimal Functioning (IZOF) model," which is designed to describe, predict, and explain the dynamics of emotion/performance for individual athletes and provide strategies for creating optimal emotional states and enhancing athletic performance (Hanin, 1999). This model marks a shift in the sport psychology literature toward a focus on the individual, but neglects the variables of social and familial contexts that have more recently been imported into the term "embodiment," along with the assertion that motor behavior is an amalgam that reveals how movement, cognition, emotion, and the social and cultural context of performance, are all intertwined (Lewthwaite and Wulf, 2010). The broader field of emotion science makes a similar claim, calling for a distinction between cognitive 
and affective consciousness. As de Gelder (2010) points out, bodily sensations are the basis for affect in humans, yet the literature on consciousness by emotions researchers tends to emphasize a cognitive awareness of emotions rather than affective awareness and feelings.

An evolutionary perspective provides us with the means to make these distinctions in ways that are perhaps particularly important for an integrative model of sport. Neuroanthropology - the study of the relation between cultural contexts and brain activity - presents a promising beginning (Campbell and Garcia, 2009; Dias, 2010; Lende and Downey, 2011). As Dias specifies, "the field of neuroanthropology has four distinct aspects: (1) exploring the interaction of brain and culture and its implication for our understanding of mind, behavior, and self; (2) examining the role of the nervous system in the creation of social and ideological structures; (3) providing empirical and critical inquiry into the interplay of neuroscience and ideologies about the brain; and (4) providing novel syntheses and advances in social science theory and the humanities that might also prove useful to brain and behavioral sciences" (Dias, 2010). A cultural neuropsychology of sport would make a contribution to this field of inquiry in specific ways.

Evolutionarily based brain substrate analysis from affective neuroscience (Panksepp, 1998, 2000, 2008, 2009), Porges' (2009, 2011) Polyvagal Theory, and Csíkszentmihályi’s (1990) conception of "flow" (completely focused motivation, single-minded immersion), all inform what I am calling the "immersive" model of sport, which combines elements from the prior two models usually used to describe human engagement with sport: the competitive and the participatory. Integrating evolutionary analysis fundamentally grounds the immersive model, and has important implications for public health and coaching strategies.

\section{AFFECT AND EVOLUTIONARY NEUROBIOLOGY}

Panksepp $(2008$, p. 59) founded the field of affective neuroscience, which studies the neural mechanisms of emotion, and claims that "all basic psychological processes are thoroughly dependent on brain biophysical processes, working in concert with body, environment, and culture." He has developed decades of empirical research to show how various affective processes, which influence behavior and action, are evolutionarily organized in the brain, and how these primary process systems interact with and influence secondary-process cognition and behavior so we can analyze interaction effects between affect and cognition - between the affective, primary processes and secondary processes associated with memory and cultural learning. Panksepp's (1998) “core emotional systems" in humans and non-human animals unite the biology of the brain with the psychology of mood and emotion. An affective neuroscience approach argues that affective states are primitive consciousness that emerges from the interaction of brain circuits that control emotional behaviors. In this sense, emotions can be conceptualized as inherited ancestral tools, and affective feelings as neurosymbolic indicators of survival values (Panksepp, 2009, p. 22).

Panksepp (1998) identifies seven core emotional systems that provide a neurobiology of affect: SEEKING, RAGE, FEAR, LUST, CARE, PANIC, and PLAY. The systems most relevant to affect in sport are SEEKING, RAGE, and PLAY, although the others are vestigially relevant as well. The SEEKING system is the appetitive motivational system that "energizes engagement with the world as individuals seek goods from the environment as well as meaning in everyday life" (Panksepp, 2009, p. 9), and is a generalized substrate for all other emotional processes. It is located in the hypothalmus and the mesolimbic dopamine system arising from the ventral tegmental area (VTA), and extends through the lateral hypothalmus to the ventral striatal nuclei, in particular the nucleus accumbens, olfactory tubercle, and further up to medial cortical regions (Panksepp, 2009).

The "PLAY" system is located within medial zones of the thalamus, and provides a safe context for young (human and nonhuman) animals to learn what they can or cannot do to each other. It can provide a physical engagement with others that is experienced as joyous, with therapeutic indications for adults, whose play urges can be re-energized by bodily activities such as dance or sport (Panksepp, 2009). The neocortex is not needed for this system, as it is not for the others, but the PLAY system has effects on the cortex, programming it to be fully social (Panksepp, 1998, 2000). In this sense, a very important function of sport is to program the brain to be social. Importantly to my purposes later, both the PLAY and SEEKING systems are activated in a context of safety.

The third core emotional system most relevant to sport is the "RAGE" system, whose expression can be linked to feelings of empowerment and assertion-activation based in a perception (real or imagined) of threat in an organism's environment. It is located in the corticomedial areas of the amygdala and descend to the medial hypothalamus via the stria terminalis, and is linked to the FEAR/PANIC system. The RAGE system is activated in response to threat, and evolutionarily speaking, being held immobile as prey (Panksepp, 2000).

\section{MODELS OF SPORT WHERE CORE EMOTIONAL SYSTEMS MANIFEST}

Historically, there have been two primary models or modalities of sport, the competitive and the participatory. The competitive model has explicit goals and quantifiable achievements, and is philosophically aligned with a mechanistic instrumentality expressed in the carefully structured, goal-directed use of time: the sport is useful for something, a means to an end. Sport practiced competitively is therefore an instrumental activity with use value, and might be psychologically linked to a sense of threat - having to overcome something in one's environment. Sport practiced in this modality most engages the SEEKING/RAGE systems. In contemporary terms, the competitive model has stood as the dominant cultural definition of "sport" in the developed world, even though those who "prefer to play for fun" represent the majority demographically speaking.

In distinction, the participatory model implements a noninstrumental modality based in play, and is practiced in the context of a sense of safety within one's group or individually. In the participatory model, there are no threats to an individual's status, whereas in the competitive model, status with a prestige hierarchy is always at stake. In the participatory mode, sport is a noninstrumental activity done for itself, not as a means to a goal/end. 
The participatory model has residual contemporary status in concepts like recreational sports leagues or "fun runs." It is directly aligned with what Panksepp defines as the PLAY system, but it also, like all systems, is activated by the SEEKING system. Sport practiced in these terms might share the goals and outcomes of affective balance therapy, or ABT, which seeks cognitive restructuring through the rebalancing of the more fundamental affective systems (Panksepp, 2009).

\section{HISTORICAL ORIGINS AND VALUES CHARACTERISTIC OF COMPETITIVE AND PARTICIPATORY MODELS}

According to sports historian Susan K. Cahn, beginning with the professionalization of physical education at the turn of the twentieth century in the US, physical educators began to advocate for a "participatory" model, which said that sport should center around the concepts of democracy, equal access, and sport for the sake of play, not victory (Cahn, 1994). This countered what physical educators saw as problems with the competitive model in that the competitive model was seen to subordinate values such as sportsmanship (consideration and respect for others), loyalty, and health to a "win-at-all-costs" mentality. The competitive model was seen to over-value ability to a degree that excluded the majority of sport participants, who were not particularly talented at sports and who enjoyed movement for movement's sake, "playing for fun." An example of the distinction between sport as practiced in competitive and participatory modes is provided by the sport of surfing, which is often split into those who participate for the pure joy of surfing ("soul" surfing) and those who compete to win surfing contests and showcase their prowess and mastery of the waves (Heywood and Montgomery, 2008).

\section{A THIRD MODEL: SPORT AS IMMERSIVE PRACTICE}

A third model of sport, what I am calling the immersive, combines elements of the competitive and participatory (Heywood, 2006, forthcoming a,b; Heywood and Montgomery, 2008). It deemphasizes those elements of the competitive model rejected by the participatory, but asserts that competition provides a number of positive effects, from in-group bonding to the empowerment of disenfranchised individuals or groups. Sport formulated as exclusively competitive or participatory excludes some of the core affective systems or forces the suppression of one to facilitate the other. The immersive model accounts for more of our core motivations, and how these inform sport participation.

Sport conceptualized as immersive practice recruits more of the core systems than does an exclusive focus on either competitive or participatory modalities. Because the SEEKING system is seen as a generalized positive appetitive motivation system, it serves as a common activation for sport practiced both competitively and in the participatory mode. While the SEEKING system is comprised of many chemical systems, the mesolimbic dopamine system is the one that provides what Panksepp (2009, p. 10) calls "the major psychobehavioral 'push." The dopamine neurons of the VTA signal us "to behave in appetitively aroused, goal-directed ways, increasingly directed toward environmental cues that predict rewards as well as dangerous situations" (Panksepp, 2009, p. 10). Dopamine and the VTA are linked to a number of "feel good" responses such as those facilitated by activities such as sex, alcohol, or drugs (Berke and Hyman, 2000; Garcia et al., 2010), and it provides one of the main reasons why sport and physical activity - practiced in whatever modality - feel good to the practitioner. Although the specific experience of "runner's high" has only been empirically linked to the opioidergic mechanisms in the frontolimbic brain areas (Boecker et al., 2008), it is clear that the SEEKING system is activated in general sport participation, perhaps particularly in the immersive modality.

A key feature of the immersive model is focused attention, the same kind of absorption seen by a hunter stalking prey or in a parent caring for an infant. The athlete experiences a sense of being "out of time," and is so focused on the movement of the body in the moment that all other considerations fall away. This model of sport includes the experience of what Mihali Csíkszentmihályi discusses as "flow" or peak experience, a kind of completely focused motivation, single-minded immersion in the activity itself (Csíkszentmihályi, 1990; Jackson and Csikszentmihalyi, 1999). An experience of "flow" is often described as performing "in the zone." The immersive model, including the experience of flow, can contain elements of the competitive model as well as participatory, but the key determinant is that the activity is performed in the context of safety, not threat/humiliation. In sport formulated in the immersive modality, the context of safety is a prerequisite. As we will see, it is around the question of safety that an athlete's social and familial contexts become crucial.

\section{INFORMING THE "IMMERSIVE SPORT" MODEL: POLYVAGAL THEORY AND THE EVOLUTIONARY QUESTION OF SAFETY}

While Panksepp's work is primarily concerned with the subcortical regions of the central nervous system (CNS), and while the SEEKING system is part of the initial CNS engagement that is directed toward environmental cues that predict reward or danger, Stephen W. Porges' work examines how evolution has modified the structures of the ANS to specifically respond to reward or danger. In his Polyvagal Theory, the mammalian ANS retains vestiges of phylogenetically older ANS, and the different levels are available as responses to environmental challenges. Emotional regulation and social behavior are thereby seen as functional derivatives of structural changes in the ANS in response to evolutionary processes.

Porges's theory argues that in mammals, an individual's ANS response to environmental challenges follows a phylogenetic hierarchy facilitated by "neuroception" - "a neural process, distinct from perception, that is capable of distinguishing environmental (and visceral) features that are safe, dangerous, or life-threatening" (Porges, 2009, p. 45). Responses start with the evolutionarily newest structures, and when all else fails, revert to the most primitive structural system. We react to real-world, environmental challenges with three neural circuits, in this order: (1) the evolutionarily newest system, the social engagement system (SES), a parasympathetic neural circuit that is expressed in the newer myelinated vagus nerve (ventral vagal complex) that promotes pro-social behavior and helps maintain calm behavioral states. (2) If the SES does not satisfy our quest for safety, we react spontaneously with the older, sympathetic nervous system that supports fight/flight behaviors. This system mediates between the two vagal circuits. (3) If fight/flight fails, and we perceive ourselves to be in 
life-threatening conditions, we resort to the oldest vagal circuit, a parasympathetic circuit expressed in the older, unmyelinated vagus nerve that inhibits motion and is linked to disassociation in response to trauma - playing dead, and having the sensation of floating out of your body (Porges, 2009, 2011).

Polyvagal Theory has important applications to the various models of sport. If the practice of sport harkens back to our evolutionary heritage as both predators and prey, immersive sport can only be practiced in a context of safety in which there are no direct needs/threats operational at the time. The competitive model is more linked to competing for resources in a context of scarcity - we compete because there is an environmental need to get resources/establish dominance, and is therefore linked to what Porges formulates as the second-level, flight/fight response: one's identity/position are under threat. Athletic dominance contains that threat, so motivations in this model are extrinsic motivation. By contrast, the participatory model of sport is linked to the first-level response of the SES, and the motivation is the activity itself practiced in a safe context. There is no need to "prove" oneself: acceptance is assumed, and the pro-social behavior of others cues the individual that the environment is safe. This is also where an individual athlete's particular social and familial contexts become important to their ability to perform. Growing up in or experiencing contexts of war or domestic violence at any age, or in households whose norms of parenting are distant and critical rather than loving and pro-social, this kind of negative experience can disrupt an athlete's affective balance long-term, thereby unsettling their neuroception and ability to dampen their flight/fight response (Panksepp, 2009; Porges, 2009, 2011) - thus blocking their ability to experience flow and reach higher levels of performance.

Similarly, Panksepp's conceptualization of “joyous" PLAY is only possible in the context of this kind of first-level response to one's environment - a context of safety where sport is not practiced as humiliation and ridicule as it can sometimes be in the competitive model. The stimulation of the PLAY system and the way that stimulation can rebalance affect is central to what Panksepp (2009, p. 21) calls ABT, which is accomplished through play as "bodily vigor, spontaneity, and creativeness of real, physical play. ... Psychiatric distress can be conceptualized as overturned tables that need to be set right, and there is unlikely to be any stronger emotional aid than that contained in the joyous potentials of PLAY." I would argue that sport practiced in the immersive modality, with focused attention, is a powerful example of this kind of PLAY.

Specific examples might include the historically recent sport of CrossFit, which incorporates a wide variety of exercises practiced in a wide variety of domains and skills, usually in a group context with emphasis on record-keeping and results (each exercise or set of exercises is timed, and past performances are compared against present). While group members are definitely competitive with each other, they are also uniformly supportive of each other, offering tips on how to better perform each skill, and continually cheering each other on and congratulating each other on performances (Crossfit.com, 2011). Similarly, the sport of running has recently undergone a tremendous influx of participation in which people run together in support of various causes (cancer research, schools), and although they might compete against each other, they are supportive of each other's performances regardless of who finishes first.

\section{TOWARD A CULTURAL NEUROPSYCHOLOGY OF IMMERSIVE SPORT}

The seven core primary process affective systems (Panksepp) provide a neurophysiological basis for understanding the models of sport that have recently been dominant. The competitive model of sport engages the SEEKING and RAGE systems, provoking a neuroception of danger, and thereby a fight/flight response seeking that has the extrinsic motivation of overcoming the threat of competitors through dominating them athletically. By contrast, the participatory model of sport engages the SEEKING and PLAY systems, triggering a neuroception of safety that facilitates a state of calm engagement and has the intrinsic motivation of participating in the activity itself, rather than overcoming rivals.

An immersive experience of sport combines elements of the participatory and competitive into a flow state-SEEKING, PLAY, and RAGE systems are all submerged into a state of calm, focused attention that is "out of time" and can facilitate the highest level of athletic performance. An immersive model of sport is evolutionarily informed in that it takes into account primal emotional systems and the way these are regulated throughout body and brain, and takes into consideration the neurological dimension of affective balance - disrupted affect due to environment/experience - that might result in a heightened fight/flight response and thus in a particular experience/internalization of particular sport norms and practices.

A cultural neuropsychology of sport - a context-dependent analysis of a specific incident, practice, or performance related to sport - would then incorporate the context of cultural support for or against sport (resistance to women's sports in certain countries; lack of infrastructure in underdeveloped countries; opportunities to play/receive training in developed countries - inflected by race, gender, class, ability, etc. - all of which would induce a neuroception of threat rather than safety). Within these broader contexts, a cultural neuropsychology of sport would also examine psychological dimensions such as an individual athlete's environment and cultural/familial context, including issues such as individual trauma response(informed by disrupted neuroception) and personality differences.

\section{CONCLUSION}

In Panksepp's and Porges' formulations, the emotions are ancestral tools for living that are regulated by the central and ANSs, and help provide ultimate level explanations of human behavior. When applied to sport, these formulations provide tremendous explanatory power to inform a comprehensive model that would examine the relationships between movement, cognition, emotion, and the social and cultural context in which movement takes place. An evolutionarily based model gives us insight into fundamental motivational systems that can then be examined in context. The contexts for sport experiences include basic sport infrastructure such as coaching, and the group dynamics facilitated by the coach.

One insight the evolutionary perspective provides is that an individual's basic assessment of safety or threat (neuroception) 
has a direct impact on movement (Porges, 2009). If the sport environment is experienced as threatening, a flow state is counterindicated, since the more primitive limbic structures responsible for fight, flight, or freeze behaviors will not be inhibited. Therefore the individual success and positive experiences associated with flow are only possible in a context of group belonging. The SES has to be engaged, and engagement is dependent upon a sense of safety that allows for focused concentration. To succeed at the highest levels, an athlete cannot be worried about jockeying for position - the fight response has to be calmed.

For the ANS to respond in the more positive ways, and for the SEEKING and PLAY systems to engage more than the RAGE system, pro-social behavior among athletes should be encouraged more than it currently is within many athletic cultures in the developed world. Starting with youth sports, coaches and media should reinvigorate the ideal of "sportsmanship" and give it real weight. Play should be encouraged more than mechanized learning and competition (and parents should be encouraged to emphasize these aspects as well). Currently, many people do not participate

\section{REFERENCES}

Berke, J. D., and Hyman, S. E. (2000). Addiction, dopamine, and the molecular mechanisms of memory. Neuron 25, 515-532.

Boecker, H., Sprenger, T., Spilker, M. E., Henriksen, G., Koppenhoefer, M., Wagner, K. J., Valet, M., Berthele, A., and Tolle, T. R. (2008). The runner's high: opioidergic mechanisms in the human brain. Cereb. Cortex 18, 2523-2531.

Cahn, S. K. (1994). Coming on Strong: Gender and Sexuality in Twentieth Century Women's Sport. Cambridge, MA: Harvard University Press.

Campbell, B. C., and Garcia, J. R. (2009). Neuroanthropology: evolution and emotional embodiment. Front. Evol. Neurosci. 1:4. doi: 10.3389/neuro.18.004.2009

Crossfit.com. (2011). What is Crossfit? Available at: http://www.crossfit. com/cf-info/what-crossfit.html

Csíkszentmihályi, M. (1990). Flow: The Psychology of Optimal Experience. New York: Harper.

de Gelder, B. (2010). The Grand challenge for frontiers in emotion science. Front. Psychol. 1:187. doi: 10.3389/fpsyg.2010.00187

Dias, A. (2010). The foundations of neuroanthropology. Front. Evol. Neurosci. 2:5. doi: 10.3389/neuro.18.005.2010

Garcia, J. R., MacKillop, J., Aller, E. L., Merriwether, A. M., Wilson, D. S., and Lum, J. K. (2010).
Associations between dopamine D4 receptor gene variation with both infidelity and sexual promiscuity. PLoS ONE 5, e14162. doi: 10.1371 /journal.pone.0014162

Hanin, Y. (ed.). (1999). Emotions in Sport. Champaign, IL: Human Kinetics.

Hanrahan, S., and Anderson, M. (2010). Routledge Handbook of Applied Sport Psychology: A Comprehensive Guide for Students and Practitioners. New York: Routledge.

Heywood, L. (2006). "Immanence, transcendence, and immersive practices: female athletes in U.S. neoliberalism," in The Scholar and the Feminist Online, Vol. 4, eds E. G. Glenney and J. Jakobsen. Available at: http://bar nard.edu/sfonline/sport/heywood_ 01.htm

Heywood, L., and Montgomery, M. (2008). "'Ambassadors of the last wilderness?': surfers, environmental ethics, and activism in America," in Tribal Play: Subcultural Journeys Through Sport, eds M. Atkinson and K. Young (Bingley: Emerald Group Publishing), 153-172.

Jackson, S. A., and Csikszentmihalyi, M. (1999). Flow in Sports. Champaign, IL: Human Kinetics.

Lende, D., and Downey, G. (2011). "About neuroanthropology," in Neuroanthropology: Understanding the Encultured Brain and Body. Available at: http://blogs. plos.org/neuroanthropology/about/

in sports because the competitive model makes them uncomfortable, and the calls to increase sport participation in order to achieve public health goals could be facilitated by more emphasis on pro-sociality. The evolutionary perspective provides more reasons why the purely results-focused, "win-at-all-costs" coaching model should be replaced by a model that creates a context of safety and cohesion that triggers each athletes' SES, thereby facilitating better performance and experience on all levels, whether competitive or participatory.

\section{ACKNOWLEDGMENTS}

The author thanks Justin R. Garcia for commentary on this article, and generally for support of the biopsychological aspects of my work, and Daniel Lende for valuable discussions concerning neuroanthropology. I also thank Glenn Geher, for enthusiastically including me in activities surrounding the NEEPS conference even through my disciplinary origins are in the humanities, and for inviting me to give the talk that was the original version of this article for his Spring 2011 EvoS Seminar Series at SUNY-New Paltz.

Lewthwaite, R., and Wulf, G. (2010). Grand challenge for movement science and sport psychology: embracing the social-cognitiveaffective-motor nature or motor behavior. Front. Psychol. 1:42. doi: 10.3389/fpsyg.2010.00042

Panksepp, J. (1998). Affective Neuroscience: The Foundations of Human and Animal Emotions. New York: Oxford.

Panksepp, J. (2000). "Emotions as natural kinds within the mammalian brain," in Handbook of Emotions, 2nd Edn, eds M. Lewis and J. M. Haviland-Jones (New York: The Guilford Press), 137-156.

Panksepp, J. (2008). “The affective brain and core consciousness: how does neural activity generate emotional feelings?" in Handbook of Emotions, 3rd Edn, eds M. Lewis, J. M. Haviland-Jones, and L. F. Barrett (New York: The Guilford Press), 47-67.

Panksepp, J. (2009). "Brain emotional systems and qualities of mental life," in The Healing Power of Emotions: Affective Neuroscience, Development, and Clinical Practice, eds D. Fosha, D. J. Siegel, and M. Solomon (New York: Norton), 1-26.

Platek, S., Geher, G., Heywood, L. Stapell, H., Porter, R. J., and Walters, T. (2011). Walking the walk to teach the talk: ancestral life strategies as the newest tool in evolutionary studies. Evol. Educ. Outreach 4, 41-51.
Porges, S. W. (2009). "Reciprocal influences between body and brain in the perception and expression of affect," in The Healing Power of Emotions: Affective Neuroscience, Development, and Clinical Practice, eds D. Fosha, D. J. Siegel, and M. Solomon (New York: Norton), 27-54.

Porges, S. W. (2011). The Polyvagal Theory: Neurophysiological Foundations of Emotions, Attachment, Communication, and Selfregulation. New York: Norton.

Conflict of Interest Statement: The author declares that the research was conducted in the absence of any commercial or financial relationships that could be construed as a potential conflict of interest.

Received: 15 July 2011; accepted: 14 October 2011; published online: 04 November 2011.

Citation: Heywood LL (2011) Affective infrastructures: toward a cultural neuropsychology of sport. Front. Evol. Neurosci. 3:4. doi: 10.3389/fnevo.2011.00004 Copyright (c) 2011 Heywood. This is an open-access article subject to a nonexclusive license between the authors and Frontiers Media SA, which permits use, distribution and reproduction in other forums, provided the original authors and source are credited and other Frontiers conditions are complied with. 